\title{
Cooperative Relay Selection in Cognitive Radio Networks
}

\author{
Tao Jing ${ }^{1}$, Shixiang Zhu ${ }^{1}$, Hongjuan $\mathrm{Li}^{2}$, Xiuzhen $\mathrm{Cheng}^{2}$, Yan $\mathrm{Huo}^{1}$ \\ ${ }^{1}$ School of Electronics and Information Engineering, Beijing Jiaotong University, Beijing, China \\ ${ }^{2}$ Department of Computer Science, The George Washington University, Washington DC, USA \\ E-mail: \{tjing,11120212\}@bjtu.edu.cn, \{hongjuan, cheng\}@gwu.edu, yhuo@bjtu.edu.cn
}

\begin{abstract}
The benefits of cognitive radio networks have been well recognized with the dramatic development of the wireless applications in recent years. While many existing works assume that the secondary transmissions are negative interference to the primary users (PUs), in this paper, we take secondary users (SUs) as positive potential cooperators for the primary users. In particular, we consider the problem of cooperative relay selection, in which the PUs actively select appropriate SUs as relay nodes to enhance their transmission performance. The most critical challenge for such a problem of cooperative relay selection is how to select a relay efficiently. But due to the potentially large number of secondary users, it is infeasible for a PU transmitter to first scan all the SUs and then pick the best one. Basically, the PU transmitter intends to observe the SUs sequentially. After observing a SU, the PU needs to make a decision on whether to terminate its observation and use the current $\mathrm{SU}$ as its relay or to skip it and observe the next SU. We address this problem by using the optimal stopping theory, and derive the optimal stopping rule. To evaluate the performance of our proposed scheme, we conduct an extensive simulation study. The results reveal the impact of different parameters on the system performance, which can be adjusted to satisfy specific system requirements.
\end{abstract}

Index Terms-Cognitive radio networks; cooperative relay selection; optimal stopping theory.

\section{INTRODUCTION}

Recently Cognitive Radio Networking has attracted a great attention from both academia and industry due to its remarkable improvement in spectrum utilization efficiency [1], [2]. The users in a cognitive radio network (CRN) are classified into two groups: primary users (PUs) and secondary users (SUs). The primary users are authorized certain licensed spectrum bands and the secondary users can sense the unused spectrum bands, which is referred to as the spectrum holes, and share them with primary users to improve the spectrum utilization.

We notice that the direct transmissions from a primary transmitter to the intended primary receiver may be severely damaged due to the unstable environment in wireless communications such as multipath fading and shadowing. Thus in this paper we consider a cooperative relaying framework in which the primary users select the secondary users that may have better channel conditions as cooperative relays to help transmit their packets. We focus on the problem of relay selection, i.e., how to efficiently find an appropriate relay node that can satisfy the primary transmitter's Quality of Service (QoS). This problem is referred to as cooperative relay selection.
The most critical concern for cooperative relay selection is efficiency, i.e., how to find out a suitable relay efficiently. As the number of secondary users could be large due to the rapidly growing number of mobile communication devices, it is impossible/impractical to scan/observe all the candidate relays for a primary transmitter. Thus we propose to apply the optimal stopping theory [3] for cooperative relay selection, with an objective of stopping early enough to avoid scanning all the candidate relays.

Our major contributions can be summarized as follows. First, we formulate the problem of cooperative relay selection as an optimal stoping problem and derive the optimal stopping rule for relay selection. Our stopping criteria considers the short term effective bit rate (instantaneous reward) and the long-term expected throughput (expected reward if all candidate relays are considered), and selects the first relay whose instantaneous reward is at least the same as the expected reward. Second, we conduct an extensive simulation study to validate the performance of our relay selection scheme. In particular, we investigate the impact of different parameters and present a thorough analysis on the results.

An overview of existing cooperative relaying techniques is provided in [4]. Various relay selection approaches [5]-[7] have been explored for cooperative relaying in general wireless networks. Some of them require channel-related information from all the candidate relay nodes, which is inefficient when the number of candidate relays is large. As a comparison, our approach does not require information from all candidate relay nodes as it scans the candidate SU relays one by one and stops when a suitable relay is identified. Relay selection is jointly considered with other network functions in [6]. Differently, our approach employs the optimal stopping theory to take into account the time to scan the candidate relays before stoping at a suitable one with a good channel quality. Optimal stopping theory has been applied to opportunistic scheduling and spectrum sensing in [8], [9]. Our work is the first one to apply stopping theory for cooperative relay selection in cognitive radio networks, to our best knowledge. It employs an optimal stopping rule to find out the relay with a good channel quality within a short observation/scan time.

The rest of this paper is organized as follows. The network model and the adopted relaying framework are illustrated in Section II. Our optimal stopping policy based cooperative relay selection scheme is detailed in Section III. The results of 
performance evaluation are reported in Section IV. And the conclusion is presented in Section V.

\section{SySTEM MODEL}

In this section, we first illustrate the system model for our problem of cooperative relay selection in cognitive radio networks. Then we introduce a simple cooperative relaying protocol adopted by this paper.

\section{A. Network Model and Assumptions}

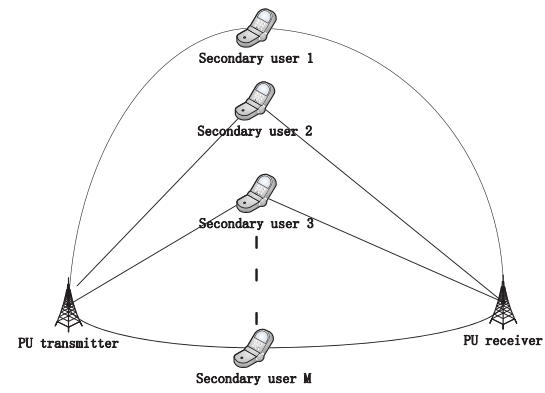

Fig. 1. Network Model.

We consider a simple time-slotted network system depicted in Fig.1. A typical primary transmitter, denoted by $P_{t}$, transmits its packets to a typical primary receiver, denoted by $P_{r}$, with the assistance from one of $M$ secondary users represented by $S_{i}, i=1,2, \cdots, M$. When $P_{t}$ needs to transmit packets to $P_{r}$, a free secondary user, which has better channel condition compared to the $P_{t}$, can be selected as a relay node by the primary user pair. The $M$ secondary users, which have the ability to help transmit packets for the primary system, are called candidate relays, and the secondary user finally selected by the primary user pair is called a cooperative relay.

It is assumed that the cooperative relay selection is performed at each time slot, and the duration of a time slot is $T$. For simplicity, we further assume that each PU pair can select at most one SU as a cooperative relay and each SU candidate relay node can only be selected by at most one PU pair. For simplicity, in this paper we first consider the network scenario where there exists only one primary pair and $M$ secondary users; then we analyze the collision probability of multiple PU pairs simultaneously selecting the same SU as a relay.

\section{B. Cooperative Relaying Protocol}

As illustrated in Fig.2, each time slot is partitioned into several components. Let $\tau$ be the time needed for observing a potential relay. We assume that $\tau$ is identical for different SUs and for different time slots. Denote by $\left\{s_{1}, s_{2}, \cdots, s_{M}\right\}$ an observation order/sequence, which is a permutation of the SU candidate relay index set $\{1,2, \cdots, M\}$. At the beginning of a time slot, $P_{t}$ starts to observe the SU candidate relay nodes sequentially according to the observation sequence. if the reward of the $k^{t h}$ observation satisfies a specific criterion, $P_{t}$ stops at the $k^{t h} \mathrm{SU}$ candidate relay node, and then delivers its packets (intended to the PU receiver $P_{r}$ ) to the secondary

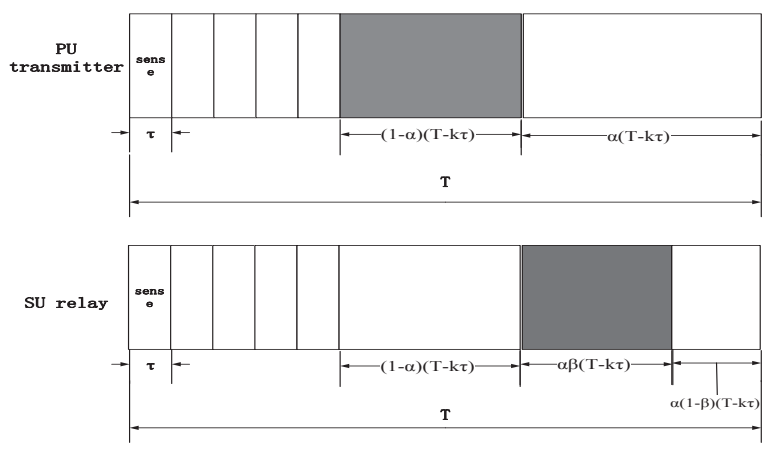

Fig. 2. The Time Slot Structure.

relay node for a fraction $(1-\alpha), 0 \leq \alpha \leq 1$, of the time $(T-k \tau)$, shown by the shadow part in the first subgraph. For the rest of the time slot $\alpha(T-k \tau), P_{t}$ transmits its data directly to $P_{r}$. The selected secondary node relays $P_{t}$ 's data over a $\beta$, $0 \leq \beta \leq 1$, fraction of $\alpha(T-k \tau)$, shown by the shadow part of the second subgraph, and then sends its own packets during the residual time. We can see that the condition $M \tau<T$ always holds. Note that a similar cooperative relaying protocol is also adopted by [10].

\section{Optimal Stopping Policy}

\section{A. Problem Formulation}

In this paper, we focus on the problem of cooperative relay selection in a CRN where a PU pair observes the SU candidate relay nodes one by one based on an observation sequence and decides whether or not to stop and select the SU node currently under observation as the cooperative relay node. To maximize the reward of the selection, the PU pair makes the decision based on the result of comparing the instantaneous reward and the expected reward of future observations. The instantaneous reward can be represented by the channel quality of the candidate relay being observed; and the expected reward of future observations is the reward the PU pair can obtain if it continues observing the following candidate relays. Therefore the relay selection problem can be formulated as a sequential decision problem and can be investigated by applying the optimal stopping theory. The stopping theory and its applications are studied in [10]. We formulate the problem of cooperative relay selection as an optimal stopping problem.

To make sure that the packets relayed by the cooperative relay node securely arrive at the destination, some conditions/restrictions should be satisfied, which can be described as follows:

$$
0<(1-\alpha) R_{p s}^{r}(t) \leq \alpha \beta R_{s p}^{s}(t)
$$

where $R_{p s}^{r}(t)$ denotes the transmission rate between the PU transmitter and the SU relay, and $R_{s p}^{s}(t)$ denotes the transmission rate between the SU relay and the PU receiver. The value of $\alpha$ is controlled by the PU transmitter. Given $\alpha$, we 
can derive the minimum value of $\beta, \beta_{\text {low }}=\frac{(1-\alpha) R_{p s}^{r}(t)}{\alpha R_{s p}^{s}(t)}$. For simplicity, it is assumed that $R_{p s}^{r}(t)=R_{s p}^{s}(t)$. Thus we have $\beta_{\text {low }}=\frac{(1-\alpha)}{\alpha}$, and $0.5 \leq \alpha \leq 1$ since $0 \leq \beta_{\text {low }} \leq 1$.

When the PU transmitter observes the channel condition of a SU, the SU returns a value for $\beta$. We know that the SU is available if the returned value is larger than or equal to $\beta_{\text {low }}$, and vice versa. Let $\theta$ denote the probability that the SU candidate relay is available. Then we define $\Theta$ as the indicator function of the availability of the SU candidate relay, which is given by

$$
\Theta= \begin{cases}0 & \text { if } \beta<\beta_{\text {low }} \text { with probabilty }(1-\theta) \\ 1 & \text { if } \beta \geq \beta_{\text {low }} \text { with probabilty } \theta .\end{cases}
$$

In order to further investigate the channel quality in our cooperative relay selection problem, we assume that the underlying channel is a flat Rayleigh fading channel, in which the instantaneous interference-plus-noise ratio (SNR) is received by the destination with an exponential distribution having a probability density function (PDF) $f(\gamma)=\frac{1}{\bar{\gamma}} e^{-\frac{\gamma}{\gamma}}$, where $\bar{\gamma}$ denotes the average SNR in the channel model. Then we can model the Rayleigh fading channel as a finite state Markov chain (FSMC) as proposed in [11]. In the FSMC we partition the SNR into $U$ intervals and then divide SNR into a finite-state space. Thus the SNR thresholds are denoted by $\Upsilon=\left\{\gamma_{1}=0, \gamma_{2}, \cdots, \gamma_{U}, \gamma_{U+1}=\infty\right\}$. If an instantaneous SNR $\Gamma$ is in $\left[\gamma_{u}, \gamma_{u+1}\right)$, the channel of the SU candidate relay is said to be in state $u$. When the PU pair observes the channel of the candidate relay, the probability of the SU being in state $s_{u}$ for the channel can be given by

$$
q_{u}=\int_{\gamma_{u}}^{\gamma_{u+1}} f(\gamma) d \gamma=e^{-\frac{\gamma_{u}}{\bar{\gamma}}}-e^{-\frac{\gamma_{u+1}}{\bar{\gamma}}}, u=1, \cdots, U
$$

Generally the achievable transmission rate is viewed as a metric for the channel quality in wireless communications. Let $r_{k}$ denote the achievable transmission rate between the PU pair and the SU candidate relay node $k$. According to the Shannon's theorem, $r_{k}$ is calculated as follows:

$$
r_{k}=W \log \left(1+\gamma_{k}\right)
$$

where $W$ denotes the bandwidth of the spectrum. Thus, the corresponding data rate, denoted as $R=\left\{r_{1}, r_{2}, \cdots, r_{U}\right\}$, can also be modeled as a discrete random variable with a distribution that is the same as the channel state

$$
\operatorname{Pr}\left\{R=r_{u}\right\}=q_{u}, u=1,2, \cdots, U
$$

The PU pair acquires the achievable transmission rate of the channel between itself and the SU candidate relay by executing the observation procedure in relay selection. The process of observation is similar to the RTS/CTS access mechanism designed for the 802.11 technique [12]. At each observation step, the PU transmitter sends a RTS (Request-To-Send) frame to the candidate relay. Upon receiving of a RTS frame, the candidate relay returns a CTS (Clear-To-Send) frame, which includes the information for calculating the achievable rate. We define $X_{k}=R_{k} \Theta$ as the valid transmission rate of the $k^{t h}$ observation step. Then the distribution of $X_{k}$ can be calculated as follows:

$$
\begin{aligned}
& p_{0}==\operatorname{Pr}\left\{X_{k}=x_{0}=0\right\}=(1-\theta) \\
& p_{u}==\operatorname{Pr}\left\{X_{k}=x_{u}=r_{u}\right\}=q_{u} \theta \\
& \text { for } 1 \leq u \leq U, 1 \leq k \leq M
\end{aligned}
$$

Then we derive the instantaneous reward function denoted by $Y_{k}$ based on the the valid transmission rate and the number of observation steps. First, we denote $c_{k}$ as a scaling factor if the PU pair stops at the $k^{t h}$ observed candidate relay node, which is given by

$$
c_{k}=1-\frac{k \tau}{T}
$$

From (7), we can see that the larger the value of $k$, the smaller the value of $c_{k}$. In other words, the more the number of SU candidate relay nodes the PU transmitter observes, the less the efficiency of the cooperative relay selection process. Therefore the payoff after the $k^{t h}$ observation attempt is given by

$$
Y_{k}=\frac{X_{k}(T-k \tau)}{k \tau+(T-k \tau)}
$$

The numerator of (8) is the number of the data bits that can be transmitted for $P_{t}$. The denominator is the total time cost for a time slot. Thus the reward $Y_{k}$ represents the average throughout the PU pair obtains at the current time slot if the pair stops after observing the $k^{\text {th }}$ SU candidate relay node and selects that node as the cooperative relay. By simplifying (8) defined above, we have :

$$
Y_{k}=c_{k} X_{k}
$$

After deriving the reward function, we summarize the optimal stopping problem in cooperative relay selection as follows: the PU pair receives the reward $Y_{k}$ after the $k^{\text {th }}$ observations. Then the PU transmitter makes a decision on whether to stop at the current candidate relay or continue to observe the next candidate relay based on the reward. Note that no recall is allowed since the channel quality is changing rapidly in cognitive radio networks due to the complicated conditions such as the mobility of the users.

\section{B. Optimal Stopping Rule}

In this subsection we intend to solve the stopping problem discussed above by deriving an optimal rule that decides when to stop observing the candidate relays. Now we derive the optimal stopping rule as the solution to the stopping problem. We formulate our solution approach as a backward induction. Denote by $V_{j}^{(M)}\left(x_{1}, x_{2}, \cdots, x_{j}\right)$ the maximum return the PU transmitter can obtain after observing the $j^{\text {th }}$ candidate relay, which is given by

$$
\begin{gathered}
V_{j}^{(M)}\left(x_{1}, x_{2}, \cdots, x_{j}\right)=\max \left\{y_{j}\left(x_{1}, x_{2}, \cdots, x_{j}\right),\right. \\
E\left\{V_{(j+1)}^{(M)}\left(x_{1}, x_{2}, \cdots, x_{j}, X_{(j+1)}\right)\right. \\
\left.\left.\mid X_{1}=x_{1}, X_{2}=x_{2}, \cdots, X_{j}=x_{j}\right\}\right\}
\end{gathered}
$$

where $y_{j}\left(x_{1}, x_{2}, \cdots, x_{j}\right)$ represents the instantaneous reward after the $k^{t h}$ observation and $E\left\{V_{(j+1)}^{(M)} \mid X_{1}=x_{1}, X_{2}=\right.$ 
$\left.x_{2}, \cdots, X_{j}=x_{j}\right\}$ represents the expected reward achieved by proceeding to observe the next candidate relay. It is optimal to stop at step $j$ if $V_{j}^{(M)}\left(x_{1}, x_{2}, \cdots, x_{j}\right)=y_{j}\left(x_{1}, x_{2}, \cdots, x_{j}\right)$, and to continue observing the candidate relays otherwise.

To better understand the backward induction, we define $Z_{M-j}$ to be the expected reward $E\left\{V_{(j+1)}^{(M)}\right\}$. That is:

$$
\begin{aligned}
Z_{M-j}= & E\left\{V_{(j+1)}^{(M)}\left(x_{1}, x_{2}, \cdots, x_{j}, X_{(j+1)} ;\right)\right. \\
& \left.\left.\mid X_{1}=x_{1}, X_{2}=x_{2}, \cdots, X_{j}=x_{j} ;\right\}\right\} .
\end{aligned}
$$

The channels of different SU candidate relays are mutually independent since the SUs behave independently. Thus the set $\left\{X_{1}, X_{2}, \cdots, X_{M}\right\}$ for the $\mathrm{SU}$ candidate relays are also mutually independent, indicating that $V_{j}^{(M)}$ only depends on $X_{j}$ and $Z_{M-j}$. Thus we can conclude that $Z_{M-j}$ is a constant that only depends on $M-j$, the remaining number of steps to continue. Note that $Z_{j}$ can be computed as follows:

$$
\begin{aligned}
& Z_{o}=-\infty \\
& Z_{1}=E\left[Y_{M}\right]=E\left[c_{M} X_{M}\right]=c_{M} \sum_{k=0}^{U} x_{k} p_{k}
\end{aligned}
$$

and for $j \geq 1$,

$$
\begin{aligned}
Z_{j+1} & =E \max \left\{Y_{M-j}, Z_{j}\right\} \\
& =\sum_{m} c_{M-j} x_{m} p_{m}+\sum_{n} Z_{j} p_{n}
\end{aligned}
$$

where $m \in\left\{k \mid c_{M-j} x_{k}>Z_{j}, k=0,1,2, \cdots, U\right\}, n \in\{k \mid$ $\left.c_{M-j} x_{k}<Z_{j}, k=0,1,2, \cdots, U\right\}$, subject to

$$
\left\{\begin{array}{l}
0 \leq m \leq U \\
0 \leq n \leq U \\
m+n=U
\end{array}\right.
$$

\section{Algorithm III-B: The Optimal Stopping Rule}

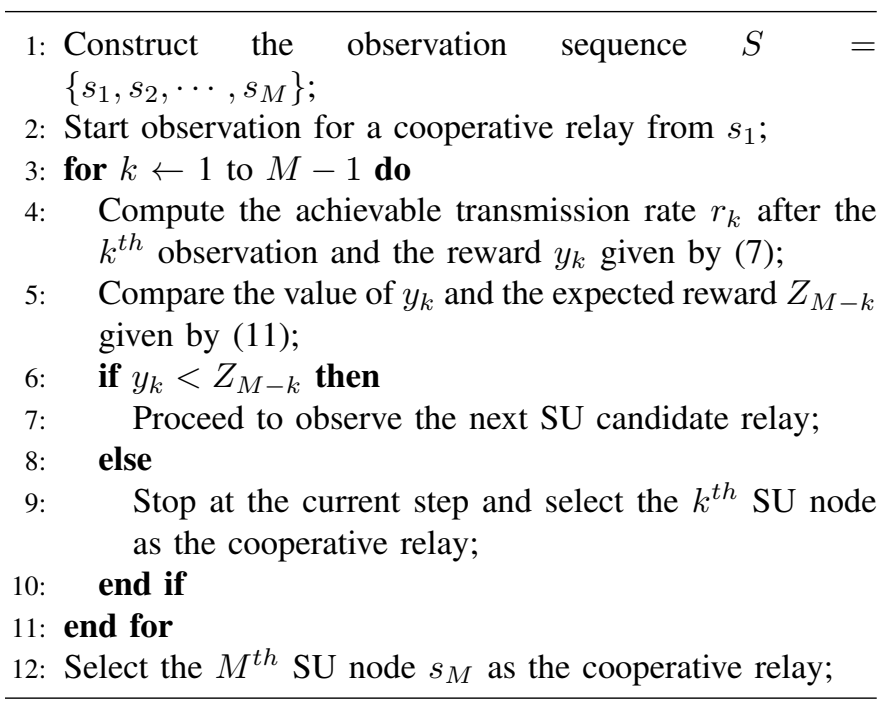

The description of the optimal stopping rule is presented in Algorithm 1. The PU pair observes a SU candidate relay node according to the observation sequence $S$, and obtains an instantaneous reward $y_{k}$ after the $k^{\text {th }}$ observation (line 4). Then the PU pair compares the value of $y_{k}$ with the value of $Z_{M-k}$ and decides to stop at step $k$ if $y_{k}>Z_{M-k}$, and to continue observing the next SU candidate relay node otherwise (line 5 to 9). Note that if the PU pair observes the last SU candidate relay in the observation sequence (that means the first $M-1 \mathrm{SU}$ candidate relays do not satisfy the quality requirement of the PU pair), it has to select the $M^{\text {th }} \mathrm{SU}$ node as the cooperative relay regardless of the value of the instantaneous reward $y_{M}$ (line 12), which is the worst case of relay selection.

\section{Performance Evaluation}

In this section we evaluate the performance of our proposed optimal stopping policy by conducting extensive simulation study. It is assumed that the duration of a time slot in our system is $0.2 \mathrm{~ms}$. And the achievable transmission rate $R_{k}$ of the $k^{\text {th }} \mathrm{SU}$ candidate relay does not change within one slot. We divide the finite-state space of SNR received by the receiver into $U=20$ intervals. The average SNR $\bar{\gamma}$ in the Rayleigh fading channel model is set to be $30 \mathrm{~dB}$. The bandwidth $W$ is set to be $1 \mathrm{MHz}$. The numerical results reported in this section are averaged over 100 independent runs.

To provide a deep insight into the optimal stopping problem in SU relay selection, we study the impacts of the observation duration $\tau$ and the parameter $\alpha$ on the system performance in terms of the number of observation steps and the average reward for the PU pair.

\section{A. The Impact of Observation Duration $\tau$}

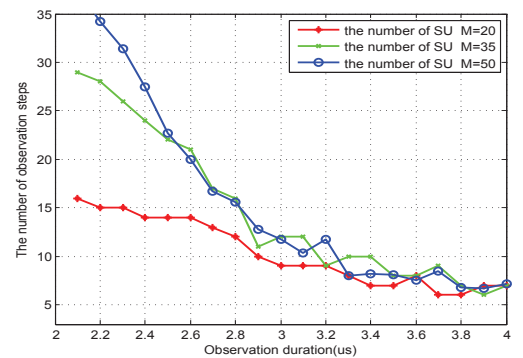

Fig. 3. The number of observation steps vs. the observation duration.

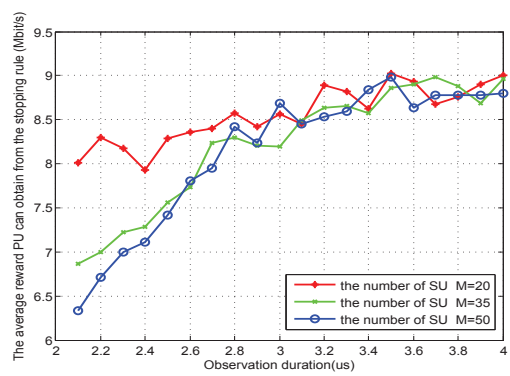

Fig. 4. The average reward for the PU pair vs. the observation duration. 
In this subsection we set the fraction $\alpha$ to be 0.8 . In Figures 3-4, we consider different metrics under different network scales. Apparently, the number of SU candidate relays $M$ influences the relay selection performance. Figure 3 shows that the number of observation steps decreases with the increase of the time duration needed for each observation. This is because the value of $\tau$ represents the cost of observing one SU candidate relay, the PU needs to stop observation as soon as possible to avoid generating a large cost. On the contrary, when the cost for observation is low, the PU tends to observe more SUs to find a better cooperative relay. We also notice that the number of observation steps is larger with a larger network scale, since the PU pair is provided with more choices when the number of the candidate relays is larger. One important common feature among the Figures 3-4 is that the three curves for three different network scales start to converge when the observation duration becomes large enough, for example, $\tau>2.7 \mu \mathrm{s}$.

From Figure 4, we can see that the average reward obtained by a PU pair increases with the time duration for each observation. Jointly considering the relationship between the observation duration and the number of observation steps, we reach the following conclusion that the less the number of observation steps, the larger the average reward a PU pair can obtain. This fact motivates the PU pair to construct a more efficient observation order and find the optimal cooperative relay as soon as possible. Note that when the observation duration increases up to $3 \mu \mathrm{s}$ or more, the number of observation steps and the average reward tend to reach a steady range. This implies the proposed optimal stopping policy results in a steady state of the relay selection system.

\section{B. The Impact of The Parameter $\alpha$}

To further investigate the availability of the SU candidate relay, we try to evaluate the system performance under the situation where the parameter $\alpha$ changes regularly. The relationship between the parameter $\alpha$ with the availability of the SU candidate relay can be obtained in Section II-B.

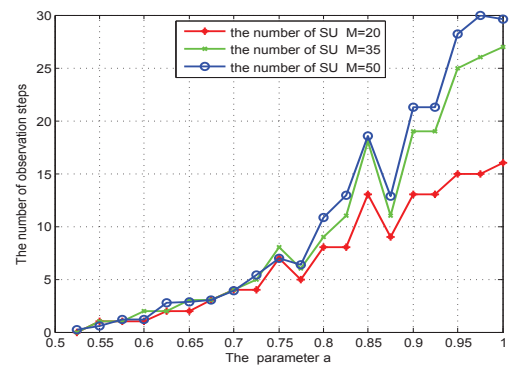

Fig. 5. The number of observation steps vs. the parameter $\alpha$.

This relationship can be found in Figure 5. A larger $\alpha$, which implies higher availability of a SU candidate relay, results in more SU candidate relays that can help the PU pair transmit packets. This leads to the fact that a PU pair is willing to spend more time to find a better SU candidate relay.

\section{CONClusion}

In this paper, we propose an optimal stopping policy to solve the problem of cooperative relay selection in cognitive radio networks. In our system, the PU pair observes the SU candidate relays orderly and selects one SU node as the cooperative relay if the transmission requirement is satisfied by this SU. We formulate this framework as an optimal stopping problem. Firstly we prove the existence of the optimal solution to the stopping problem. Then we derive an optimal stopping rule to find the optimal solution. Extensive numerical simulation is performed to study the impact of different parameters on the system performance.

In our future research, we plan to jointly consider relay selection and channel assignment to enhance the dynamic spectrum access efficiency. Moreover, we intend to extend our current investigation to the problem of selecting multiple relays (single-hop or multi-hop) for each primary transmitter.

\section{ACKNOWLEDGMENT}

The authors would like to thank the support from the National Natural Science Foundation of China (Grant No. 61272503, 61272505 and 61172074), the Fundamental Research Funds for the Central Universities of China (2012YJS007), and the National Science Foundation of the US (CNS-1162057).

\section{REFERENCES}

[1] W. Li, X. Cheng, T. Jing, Y. Cui, K. Xing, and W. Wang, "Spectrum assignment and sharing for delay minimization in multi-hop multi-flow crns," IEEE Journal on Selected Areas in Communications (JSAC), Special Issue on Cognitive Radio, March 2013.

[2] X. Xing, T. Jing, Y. Huo, H. Li, and X. Cheng, "Channel quality prediction based on bayesian inference in cognitive radio networks," in IEEE INFOCOM, 2013.

[3] T. Ferguson. Optimal stopping and applications. [Online]. Available: http://doi.acm.org/10.1145/1614320.1614325

[4] W. Elmenreich, N. Marchenko, H. Adam, C. Hofbauer, G. Brandner, C. Bettstetter, and M. Huemer, "Building blocks of cooperative relaying in wireless systems," Elektrotechnik und Informationstechnik, vol. 125, pp. 353-359, 2008, 10.1007/s00502-008-0571-7. [Online]. Available: http://dx.doi.org/10.1007/s00502-008-0571-7

[5] J. Jia, J. Zhang, and Q. Zhang, "Cooperative relay for cognitive radio networks," in IEEE INFOCOM, April 2009, pp. $2304-2312$.

[6] B. Wang, Z. Han, and K. Liu, "Distributed relay selection and power control for multiuser cooperative communication networks using buyer/seller game," in IEEE INFOCOM, May 2007, pp. $544-552$.

[7] W. Li, X. Cheng, T. Jing, and X. Xing, "Cooperative multi-hop relaying via network formation games in cognitive radio networks," in IEEE INFOCOM, 2013

[8] D. Zheng, W. Ge, and J. Zhang, "Distributed opportunistic scheduling for ad-hoc communications: An optimal stopping approach," in $A C M$ MobiHoc, September 2007, pp. 1-10.

[9] T. Shu and M. Krunz, "Throughput-efficient sequential channel sensing and probing in cognitive radio networks under sensing errors," in ACM Mobicom, September 2009, pp. 37-48.

[10] K. Khalil, M. Karaca, O. Ercetin, and E. Ekici, "Optimal scheduling in cooperate-to-join cognitive radio networks," in IEEE INFOCOM, April 2011, pp. $3002-3010$.

[11] H. S. Wang and N. Moayeri, "Finite-state markov channel-a useful model for radio communication channels," IEEE Transactions on Vehicular Technology, vol. 44, no. 1, pp. 163 -171, Feb 1995.

[12] G. Bianchi, "Performance analysis of the ieee 802.11 distributed coordination function," IEEE Journal on Selected Areas in Communications, vol. 18 , no. 3, pp. $535-547$, March 2000. 